\title{
Association between intrahepatic cholestasis in pregnancy and gestational diabetes mellitus. A retrospective analysis
}

\author{
Agata Majewska, Bartosz Godek, Dorota Bomba-Opon, Miroslaw Wielgos \\ $1^{\text {st }}$ Department of Obstetrics and Gynecology, Medical University of Warsaw, Poland
}

\begin{abstract}
Objectives: Intrahepatic cholestasis of pregnancy (ICP) is a liver specific disorder affecting $0.08 \%-27.6 \%$ pregnant women. It is characterized by reduced expression of the primary bile acid farnesoid receptor (FXR). In recent studies, it has been showed that FXR has an impact on normal glucose homeostasis. Based on that it was suggested that the level of bile acids correlates with glucose level. The aim of the study was to evaluate the association between ICP and gestational diabetes mellitus (GDM).

Material and methods: 102 singleton patients complicated by ICP were included to the study and divided into two groups: non-GDM group (74 patients) and GDM group (28 patients). ICP was diagnosed based on the serum bile acids level $>10 \mu \mathrm{mol} / \mathrm{L}$ and GDM with the $75 \mathrm{~g}$ oral glucose tolerance test and FIGO guidelines. Demographic and clinical outcome data (including maternal age, BMI and infant weight) and ICP and GDM biochemical markers were collected.

Results: The incidence of GDM in ICP patients was $27.45 \% .73 \%$ of women included to the study developed mild cholestasis. Lower levels of serum bile acids were correlated with GDM group. When compared mean total bilirubin level was significantly higher in non-GDM group. Transaminases (ALT, AST) and neonate condition including mean birth weight revealed no significant difference between the groups. On the other hand, prevalence of large for gestational age was significantly higher in non-GDM group $(\mathrm{p}<0.00001)$.
\end{abstract}

Conclusions: The incidence of ICP is higher in women with GDM.

Key words: gestational diabetes mellitus; intrahepatic cholestasis of pregnancy; bile acids; preterm delivery

Ginekologia Polska 2019; 90, 8: 458-463

\section{INTRODUCTION}

Intrahepatic cholestasis of pregnancy (ICP), also known as obstetric cholestasis, is a liver specific disorder associated with elevated serum bile acids, liver function tests, pruritus and increased rates of adverse fetal outcomes. The incidence of ICP depends on demographic variation affecting 0.08$27.6 \%$ pregnant women. The highest rate is seen in South America in Chile (11.8-27.6\%) [1]. In Poland, estimated rate of ICP is $1.5 \%$ but is based on few studies on small population.

Gestational diabetes mellitus (GDM) is a disorder diagnosed based on hyperglycemia that is recognized for the first time during pregnancy. The rate of pregnant women developing the condition varies between 1-14\% and is higher in Asian than in Caucasian ethnicity [2,3]. There are few risk factors of the development of GDM, for example obesity, age above 35 years old and family history of T2DM [2]. It has been showed that
GDM has an impact on the outcome in both mother and fetus. It predisposes the neonate to macrosomia, hypoglycemia, hyperbilirubinemia, polycythemia, respiratory disorders, shoulder dystocia and in the future to obesity and ultimately diabetes. The prevalence of the disorder is increasing and it correlates with progressive rising of obesity and T2DM cases in population [4].

The direct cause of correlation between ICP and GDM is unknown. There is increasing amount of studies that show the role for the primary bile acid receptor Farnesoid $X$ receptor (FXR) in glucose and lipid levels apart from its known impact on bile acids metabolism [5]. It is suggested that during gestation if the homeostasis of one mentioned substances is dysregulated it might correlate with higher risk of abnormal levels of another. One of the prospective study of 31 patients with ICP reported a significantly higher 
blood glucose level while oral glucose tolerance testing (OGTT) [6].

Based on that it was suggested that the level of bile acids correlates with cholesterol and glucose level. Consequently, there is increasing amount of studies that ICP is associated with a higher risk of developing GDM. We decided to investigate further the correlation between these both conditions.

\section{Objectives}

The aim of this study was to investigate the association between ICP and GDM in a group of pregnant women diagnosed with ICP who undergone routing screening for GDM.

\section{MATERIALS AND METHODS}

A total group of 5676 singleton patients who gave birth at the Department of Obstetrics and Gynecology between January 2015 and December 2017 were included in the retrospective study. Intrahepatic cholestasis of pregnancy was diagnosed according to PTGiP (Polish Gynecological and Obstetrics Society) recommendations and it was based on the serum bile acids level above $>10 \mu \mathrm{mol} / \mathrm{L}$, elevated liver function tests and pruritus. After 24 weeks, universal screening for GDM was performed using the World Health Organization (WHO) $75 \mathrm{~g}$ oral glucose tolerance test (OGTT) and diagnostic criteria were based on the FIGO (The International Federation of Gynecology and Obstetrics) guidelines including: fasting blood glucose level above $92 \mathrm{mg} / \mathrm{dL} ; 75 \mathrm{~g}$ OGTT 1-hour blood glucose level of $\geq 180 \mathrm{mg} / \mathrm{dL} ; 75 \mathrm{~g}$ OGTT 2-hour blood glucose level above $153 \mathrm{mg} / \mathrm{dL}$ [7].

Based on the upon diagnostic criteria 102 out of 5676 patients registered in the clinic during the period were diagnosed with ICP. They were divided into 2 groups: non-GDM group of 74 patients diagnosed with ICP and OGTT results within the reference range and GDM group of 28 patients diagnosed with ICP and GDM.

Both groups were compared in terms of maternal age, pre-gestational BMI, pregnancy weight gain, delivery week, the method of delivery, the percentage of preterm birth and biochemical results. The groups were also compared

Table 1. The main maternal results of the study group

\begin{tabular}{|l|l|}
\hline Variable & Study group \\
\hline Vaginal delivery & $26.4 \%$ \\
\hline Cesarean section & $73.6 \%$ \\
\hline Pre-pregnancy BMI $\geq \mathbf{2 5} \mathbf{~ k g} / \mathbf{m}^{\mathbf{2}}$ [\%] & $33 \%$ \\
\hline Gestational weight gain $[\mathbf{k g}]$ & $11.84 \pm 5.33$ \\
\hline FGL & $84.35 \pm 21.94$ \\
\hline O h OGTT & $83.15 \pm 21.97$ \\
\hline 1 h OGTT & $133.78 \pm 50.32$ \\
\hline 2 h OGTT & $117.44 \pm 44.23$ \\
\hline
\end{tabular}

in terms of neonatal status assessed using the Apgar score 1 minute and 5 minutes after birth and other neonatal outcomes such as birth weight.

One-factor analysis was performed with the global significance level of 0.05 . Variables were compared by means of Student's t-test, Mann-Whitney's U-test, and chi-squared test.

\section{RESULTS}

5676 patients delivered in our hospital between 20152017. Out of these, 485 (8.54\%) developed gestational diabetes mellitus. $102(1.80 \%)$ patients were included to our study with diagnosis of intrahepatic cholestasis of pregnancy.

The main maternal results of the study group are presented in the Table 1 . The mean age of the patients was $31.89 \pm 4.84$ (range $23-44$ ) with $54 \%$ nulliparous and $46 \%$ multiparous. The mean gestational age at delivery was $255.53 \pm 15.53$ (range 236-286). 73 patients (71.6\%) delivered between 34-36 week and 6 (5.9\%) before 34 week of gestation. Most of patients (73.6\%) gave birth by cesarean section.

Figure 1 represents the prevalence of ICP in the population and in pregnancies complicated by GDM. The results showed that the incidence of ICP was 3 times higher in patients with GDM diagnosis $(p<0.0001)$. Figure 2 describes the prevalence of GDM in the population and in pregnancies

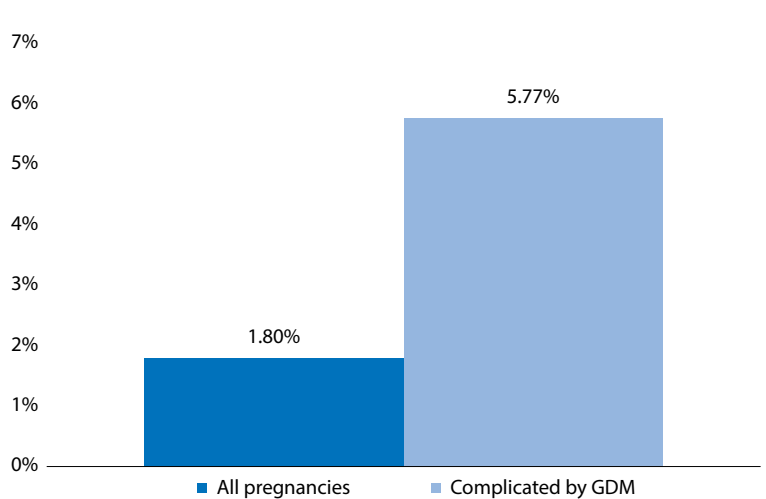

Figure 1. Prevalence of ICP in pregnancy

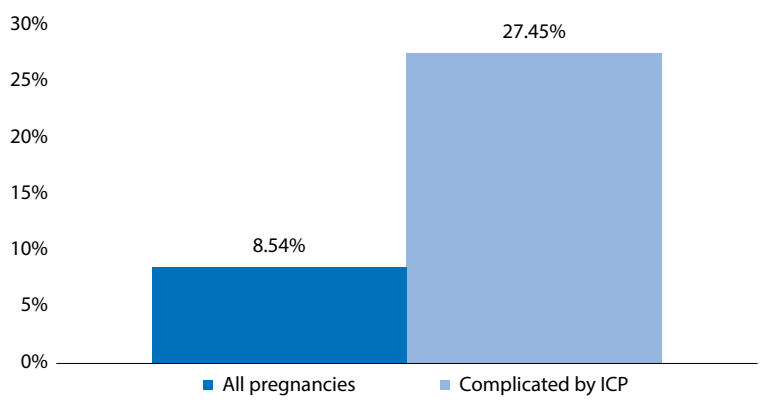

Figure 2. Prevalence of GDM in pregnancy 
complicated by ICP. The analysis revealed the prevalence of GDM tripled in a group of patients who developed ICP in comparison to all pregnancies $(p<0.0001)$.

Patients were divided into two groups based on the prevalence of GDM. In non-GDM group (74 patients) were diagnosed with ICP and had glucose level results between the reference range and in GDM group (28 patients) were diagnosed with ICP and GDM. The demographics and maternal results of the groups are presented in Table 2.

When compared, there was no significant difference in maternal age between the groups $(p=0.38)$. Patients were analyzed by gravidity and parity. The results revealed higher incidence of multigravidity and multiparity in non-GDM group than in GDM group but the difference was not statistically significant $(p>0.05)$. Maternal results showed that pre-pregnancy $\mathrm{BMI} \geq 25 \mathrm{~kg} / \mathrm{m}^{2}$ was significantly more often seen in patients in GDM group ( $p=0.039$ ). On the other hand, weight gain was observed as not significantly higher in non-GDM group than GDM group (12.19 kg vs $10.82 \mathrm{~kg}$ ).

The diagnosis of GDM was performed between 24-28 gestational age. The $75 \mathrm{~g}$ OGTT results revealed signifi- cant difference between non-GDM group and GDM group. Furthermore, the groups differed in fasting glucose level results $(p=0.002)$.

Most of our patients had preterm delivery. We divided them into 3 groups: delivery below $34 \mathrm{Hbd}$, delivery between 34-36 $\mathrm{Hbd}$ and delivery above $36 \mathrm{Hbd}$. Most of patients from both groups had delivery between $34-36 \mathrm{Hbd}$. When compared, non-GDM and GDM group did not differ significantly in amount of given births in each from above described 3 groups $(p=0.33)$.

The study group was also analyzed based on the route of delivery. In the non-GDM group rate of cesarean section was $71.2 \%$ and in the GDM group 76.9\%. The route of delivery did not differ significantly between the two groups ( $p=0.396)$. Based on our results gestational diabetes mellitus had no significant impact on the route of delivery in patients diagnosed with ICP.

Laboratory liver function tests results of the study group are presented in the Table 3 . The study revealed that bile acids were not statistically higher in non-GDM group than in GDM group (34.27 vs 25.86;

\begin{tabular}{|c|c|c|c|}
\hline Variable & $\begin{array}{l}\text { Non-GDM group } \\
\text { (74 patients) }\end{array}$ & $\begin{array}{l}\text { GDM group } \\
\text { (28 patients) }\end{array}$ & p value \\
\hline Age [years] & $31.51 \pm 4.5$ & $32.42 \pm 4.56$ & 0.38 \\
\hline Multigravida [\%] & $60 \%$ & $46.15 \%$ & 0.22 \\
\hline Multiparous [\%] & $50 \%$ & $39.29 \%$ & 0.34 \\
\hline Pre-pregnancy BMI $\geq 25 \mathrm{~kg} / \mathrm{m}^{2}[\%]$ & $24,32 \%$ & $50 \%$ & 0.039 \\
\hline Gestational weight gain [kg] & $12.19 \pm 4.91$ & $10.82 \pm 6.34$ & 0.26 \\
\hline FGL & $81.91 \pm 8.04$ & $90.84 \pm 18.53$ & 0.002 \\
\hline Oh OGTT & $79.72 \pm 6.72$ & $91.96 \pm 19.6$ & 0.005 \\
\hline 1h OGTT & $118.57 \pm 25.37$ & $175.28 \pm 45.77$ & 0.00002 \\
\hline 2h OGTT & $103.55 \pm 22.98$ & $154.28 \pm 37.97$ & 0.00001 \\
\hline Gestational age & $252.6 \pm 15.6$ & $251.0 \pm 17.2$ & 0.74 \\
\hline Delivery < $34 \mathrm{Hbd}$ & $8.1 \%$ & $0 \%$ & 0.33 \\
\hline Delivery 34-36 Hbd & $68.9 \%$ & $78.6 \%$ & 0.33 \\
\hline Delivery > $36 \mathrm{Hbd}$ & $23 \%$ & $21.4 \%$ & 0.33 \\
\hline Vaginal delivery & $28.8 \%$ & $23.1 \%$ & 0.396 \\
\hline Cesarean section & $71.2 \%$ & $76.9 \%$ & 0.396 \\
\hline Birth weight [g] & $2899.9 \pm 617.2$ & $2885.8 \pm 736.93$ & 0.08 \\
\hline
\end{tabular}

Table 3. Laboratory liver function tests

\begin{tabular}{|l|l|l|l|}
\hline Variable & $\begin{array}{l}\text { Non-GDM group } \\
\text { (74 patients) }\end{array}$ & $\begin{array}{l}\text { GDM group } \\
\text { (28 patients) }\end{array}$ & p value \\
\hline Total bilirubin level & $1.04 \pm 2.13$ & $0.78 \pm 1.36$ & 0.013 \\
\hline ALT [range] & $6-1031$ & $13-475$ & 0.27 \\
\hline AST [range] & $14-469$ & $29-201$ & 0.12 \\
\hline Bile acids $<40 \mathrm{umol} / \mathrm{L}$ & $71.2 \%$ & $79.2 \%$ & 0.73 \\
\hline
\end{tabular}




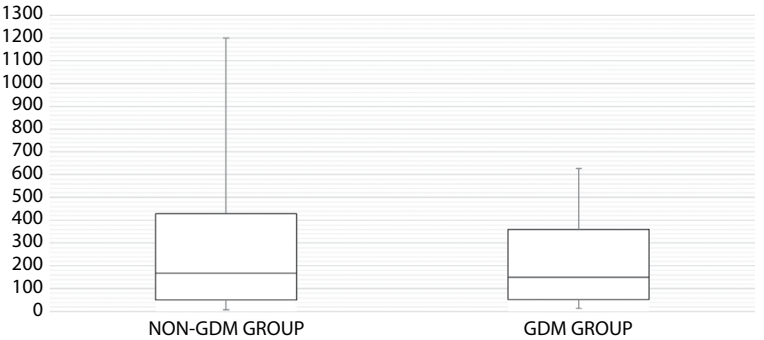

Figure 3. Alanine aminotransferase results, mean \pm SEM

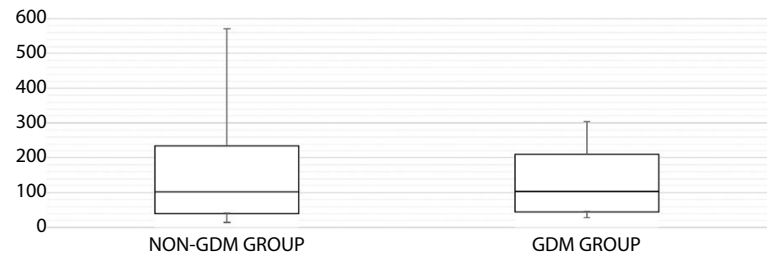

Figure 4. Asparagine aminotransferase results, mean \pm SEM

$\mathrm{p}=0.33)$. Similarly, the transaminases mean results (ALT and AST) showed no significant difference between non-GDM and GDM group (ALT: 194.35 vs 147.77, $p=0.27 ;$ AST: 110.2 vs 78.07, $p=0.12$ ). Figure 3 and 4 represent the results of ALT and AST describing mean and SEM in both groups. When the aminotransferases results were compared, it was observed wide range between the lowest and the highest rate of both ALT and AST in both groups included to the study.

As shown on Figure 5 most of patients included to the study $(73 \%)$ developed mild cholestasis with bile acids below $40 \mathrm{umol} / \mathrm{L}$. When the severity of cholestasis was compared, the rate of mild cholestasis was $71.2 \%$ in non-GDM group an $79.2 \%$ in GDM group ( $p>0.05$ ). It was analyzed whether the levels of the serum bile acids have an impact on the prevalence and severity of GDM. The Figure 6 reveals the higher prevalence of GDM correlated with lower levels of serum bile acids. GDM grade 2 developed in patients only in the group with bile acids below 40 umol/L (4.55\% of patients diagnosed with mild ICP developed GDMG2). On the other hand, analysis showed that the difference was not statistically significant $(p=0.73)$. When compared mean total bilirubin level was significantly higher in nonGDM group compared to GDM group.

The neonate's results showed no significant difference in birth weight between the groups $(p=0.08)$. The prevalence of SGA was observed in $13.48 \%$ and LGA in $34.83 \%$ of neonates analyzed in the study. When compared it was statistically higher prevalence of small for gestational age in GDM group ( $p<0.00001)$. Large for gestational age was statistically higher in non-GDM group than GDM group, $36.92 \%$ and $29.17 \%$ respectively $(p<0.00001)$.

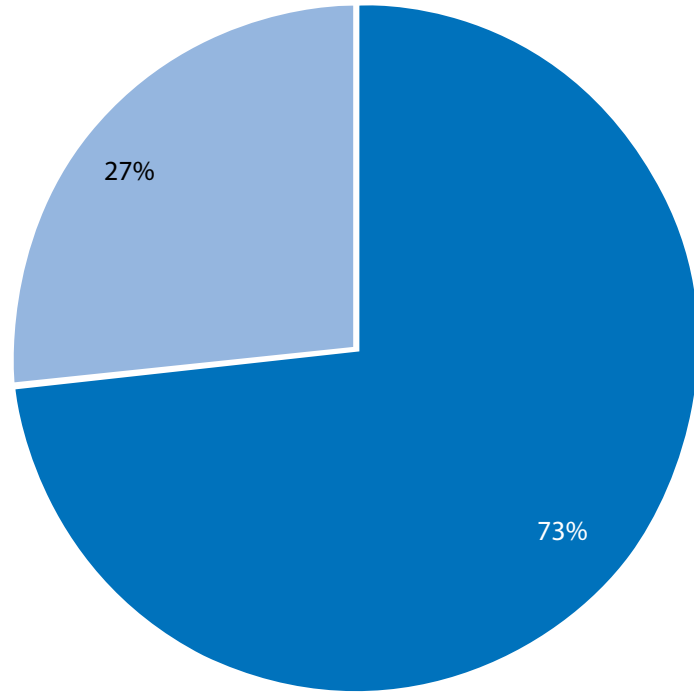

- below $<40 \mathrm{umol} / \mathrm{L}$ above $>40 \mathrm{umol} / \mathrm{L}$

Figure 5. Prevalence of mild and severe intrahepatic cholestasis in pregnancy

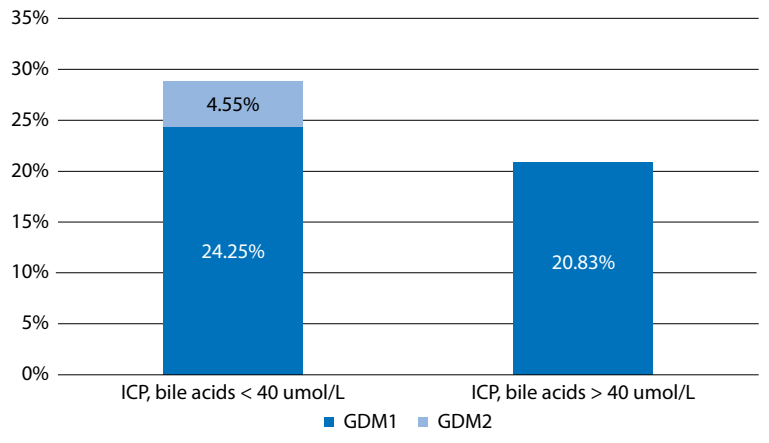

Figure 6. Prevalence of GDM in mild and severe cholestasis

\section{DISCUSSION}

Nowadays Pathology of Pregnancy is an increasing problem worldwide. It is connected to sedentary lifestyle that has an impact on higher rate of obesity, diabetes and hypertension.

There are many studies that confirm that weight before pregnancy has an impact on both fetus and pregnant woman. [8-11]. In our study, almost one quarter of patients with ICP and half of patients with ICP and GDM had BMI before pregnancy $\geq 25 \mathrm{~kg} / \mathrm{m}^{2}$. The difference between the groups was statistically different. Thus, it might be taken to account weight loss before pregnancy prevents complications of pregnancy in some patients from the risk group $[12,13]$.

Most of our patients had preterm delivery. Our study revealed that most of our patients gave birth between 34 and 36 week of gestation. According to the literature, it seems 
that GDM increases risk of preterm delivery [14]. Interestingly, in our study there was no statistical difference in number of preterm deliveries between GDM and non-GDM group. It might be correlated with the effective treatment of GDM in the hospital as other studies suggest [15].

Preterm delivery increases rate of cesarean sections. As other studies show GDM increases risk of CC instead of vaginal delivery in comparison to general population of pregnant women [16-18]. In our study the incidence of CC was over $70 \%$ percent in both studied groups. Interestingly, we found that having more than one pathology of pregnancy did not have significant impact on the route of delivery.

Another problem that shows our study is age of women deciding for pregnancy. As our results revealed mean age of our study group was above 30 years old. It has an impact on both fetus and pregnant patients leading to increased risk of pathology of pregnancy. It has been proven that age above 35 years old is a risk factor of gestational diabetes [19]. Changing habits of population may lead in the nearest future to increased rate of this condition. Based on our results and other studies it may also have an impact on rate of intrahepatic cholestasis of pregnancy $[6,20]$.

Few studies revealed that bile acids metabolism correlates with insulin resistance. It is thought, that bile acids are involved in the regulation of hepatic glucose metabolism by FXR-mediated pathways [21, 22]. Based on that some studies searched whether ICP might increase risk of GDM [20]. Martineau et al. evaluated the incidence of GDM in ICP patients is higher than in general population and the rate by then is $13.6 \%$ [23]. Our study confirmed the thesis. We received higher proportion of the patients in our study (29.94\%). It might relate to smaller study group included in our research work than in the mentioned article (57 724 vs 5676 patients). Furthermore $1^{\text {st }}$ Department of Obstetrics and Gynecology, Medical University of Warsaw is Grade III referral Hospital and consequently it admits a higher rate of patients with pathology of pregnancy than is seen in general population.

In our results ALT are higher than AST values in patients with ICP. ALT is thought to be more sensitive marker of ICP [1]. In one of the studies it was analyzed that the increase of ALT is 2-10-fold in serum levels than the rise of ALT [24].

The biggest advantage of this research was the uniform inclusion criteria of patients to the study. The other advantage is small amount of missing data. There are also few limitations of the study. First, it was conducted in Clinic with Pathology Ward, that might have an impact on rate and severity of both GDM and ICP. The glycaemia and bile acids level might be slightly higher than in general in patients with upon mentioned diagnoses as it is III Range of Reference Hospital admitting also the most severe cases. Secondly, as it was conducted in Warsaw it does not impress general population in Poland. It should be compared with the studies from smaller cities to have the point of view how both conditions correlate in general in Poland. Lastly, the study group was a small population and that might had an impact on not statistically different results in the study between the groups and based on that to not make definitive conclusions.

\section{CONCLUSIONS}

We found that ICP correlates with impaired glucose tolerance. These findings might have an impact on healthcare of the fetus and pregnant women with diagnosis of one of the mentioned conditions. The results of the earlier studies are the evidence supporting the thesis about association between ICP and GDM. It might be taken into an account a consideration of screening for glucose intolerance in patients with diagnosis of new-onset cholestasis. To make clarify the steps regarding mentioned test more studies are required. It will help to find out the direct reason responsible for higher rate of coincidence of the upon two conditions. Consequently, it may have role in better care of the pregnant woman and fetal well-being.

\section{REFERENCES}

1. Geenes V, Williamson C. Intrahepatic cholestasis of pregnancy. World J Gastroenterol. 2009; 15(17): 2049-2066, doi: 10.3748/wjg.15.2049, indexed in Pubmed: 19418576.

2. Bain E, Crane M, Tieu J, et al. Diet and exercise interventions for preventing gestational diabetes mellitus. Cochrane Database Syst Rev. 2015(4): CD010443, doi: 10.1002/14651858.CD010443.pub2, indexed in Pubmed: 25864059.

3. Wan $C S$, Abell $S$, Aroni $R$, et al. Ethnic differences in prevalence, risk factors, and perinatal outcomes of gestational diabetes mellitus: A comparison between immigrant ethnic Chinese women and Australian-born Caucasian women in Australia. J Diabetes. 2019 [Epub ahead of print], doi: 10.1111/1753-0407.12909, indexed in Pubmed: 30773821.

4. Mirghani Dirar A, Doupis J. Gestational diabetes from A to Z. World J Diabetes. 2017; 8(12): 489-511, doi: 10.4239/wjd.v8.i12.489, indexed in Pubmed: 29290922.

5. Abu-Hayyeh S, Papacleovoulou G, Lövgren-Sandblom A, et al. Intrahepatic cholestasis of pregnancy levels of sulfated progesterone metabolites inhibit farnesoid X receptor resulting in a cholestatic phenotype. Hepatology. 2013; 57(2): 716-726, doi: 10.1002/hep.26055, indexed in Pubmed: 22961653.

6. Martineau MG, Raker C, Dixon $\mathrm{PH}$, et al. The metabolic profile of intrahepatic cholestasis of pregnancy is associated with impaired glucose tolerance, dyslipidemia, and increased fetal growth. Diabetes Care. 2015; 38(2): 243-248, doi: 10.2337/dc14-2143, indexed in Pubmed: 25504029.

7. Zaręba-Szczudlik J, Pykało-Gawińska D, Stępień A, et al. Gestational diabetes mellitus (GDM) - do the number of fulfilled diagnostic criteria predict the perinatal outcome? Ginekol Pol. 2018; 89(7): 381-387, doi: 10.5603/GP.a2018.0065, indexed in Pubmed: 30091448.

8. Moftakhar L, Solaymani-Dodaran M, Cheraghian B. Role of obesity in gestational hypertension in primigravidae women: A case control study in Shadegan, Iran. Med J Islam Repub Iran. 2018; 32: 104, doi: 10.14196/mjiri.32.104, indexed in Pubmed: 30815399.

9. Nowak $M$, Kalwa $M$, Oleksy $P$, et al. The relationship between pre-pregnancy $\mathrm{BMI}$, gestational weight gain and neonatal birth weight: a retrospective cohort study. Ginekol Pol. 2019; 90(1): 50-54, doi: 10.5603/GP.2019.0008, indexed in Pubmed: 30756371.

10. Catalano PM, Shankar K. Obesity and pregnancy: mechanisms of short term and long term adverse consequences for mother and child. BMJ. 2017; 356: j1, doi: 10.1136/bmj.j1, indexed in Pubmed: 28179267.

11. Najafi F, Hasani J, Izadi N, et al. The effect of prepregnancy body mass index on the risk of gestational diabetes mellitus: A systematic review and dose-response meta-analysis. Obes Rev. 2019; 20(3): 472-486, doi: 10.1111/obr.12803, indexed in Pubmed: 30536891 
12. Radesky JS, Oken E, Rifas-Shiman SL, et al. Diet during early pregnancy and development of gestational diabetes. Paediatr Perinat Epidemiol. 2008;22(1): 47-59, doi: 10.1111/j.1365-3016.2007.00899.x, indexed in Pubmed: 18173784.

13. Forsum E, Brantsæter AL, Olafsdottir AS, et al. Weight loss before conception: A systematic literature review. Food Nutr Res. 2013; 57, doi: 10.3402/fnr.v57i0.20522, indexed in Pubmed: 23503117.

14. YogevY, Langer O. Spontaneous preterm delivery and gestational diabetes: the impact of glycemic control. Arch Gynecol Obstet. 2007; 276(4):361-365, doi: 10.1007/s00404-007-0359-8, indexed in Pubmed: 17429669.

15. Bar-Hava I, Barnhard Y, Scarpelli SA, et al. Gestational diabetes and preterm labour: is glycaemic control a contributing factor?. Eur J Obstet Gynecol Reprod Biol. 1997; 73(2): 111-114, doi: 10.1016/s03012115(97)02707-3, indexed in Pubmed: 9228488.

16. Mylonas I, Friese K. Indications for and Risks of Elective Cesarean Section. Dtsch Arztebl Int. 2015; 112(29-30): 489-495, doi: 10.3238/arztebl.2015.0489, indexed in Pubmed: 26249251

17. Bacq $Y$, Sentilhes L. Intrahepatic cholestasis of pregnancy: Diagnosis and management. Clin Liver Dis (Hoboken). 2014; 4(3): 58-61, doi: 10.1002/cld.398, indexed in Pubmed: 30992922.

18. Grabowska K, Stapińska-Syniec A, Saletra A, et al. Labour in women with gestational diabetes mellitus. Ginekol Pol. 2017; 88(2): 81-86, doi: 10.5603/GP.a2017.0016, indexed in Pubmed: 28326517.
19. Cypryk K, Szymczak W, Czupryniak L, et al. Gestational diabetes mellitus - an analysis of risk factors. Endokrynol Pol. 2008; 59(5): 393-397, indexed in Pubmed: 18979449.

20. Gao J, Xu B, Zhang X, et al. Association between serum bile acid profiles and gestational diabetes mellitus: A targeted metabolomics study. Clin Chim Acta. 2016; 459: 63-72, doi: 10.1016/j.cca.2016.05.026, indexed in Pubmed: 27246871.

21. Prawitt J, Caron S, Staels B. Bile acid metabolism and the pathogenesis of type 2 diabetes. Curr Diab Rep. 2011; 11(3): 160-166, doi: 10.1007/s11892-011-0187-x, indexed in Pubmed: 21431855.

22. Trauner $M$, Claudel $T$, Fickert $P$, et al. Bile acids as regulators of hepatic lipid and glucose metabolism. Dig Dis. 2010; 28(1): 220-224, doi: 10.1159/000282091, indexed in Pubmed: 20460915.

23. Martineau M, Raker C, Powrie R, et al. Intrahepatic cholestasis of pregnancy is associated with an increased risk of gestational diabetes. Eur J Obstet Gynecol Reprod Biol. 2014; 176: 80-85, doi: 10.1016/j. ejogrb.2013.12.037, indexed in Pubmed: 24462052.

24. Fisk N, Bye W, Storey G. Maternal Features of Obstetric Cholestasis: 20 Years Experience at King George V Hospital. The Australian and New Zealand Journal of Obstetrics and Gynaecology. 1988; 28(3): 172-176, doi: 10.1111/j.1479-828x.1988.tb01657.x. 